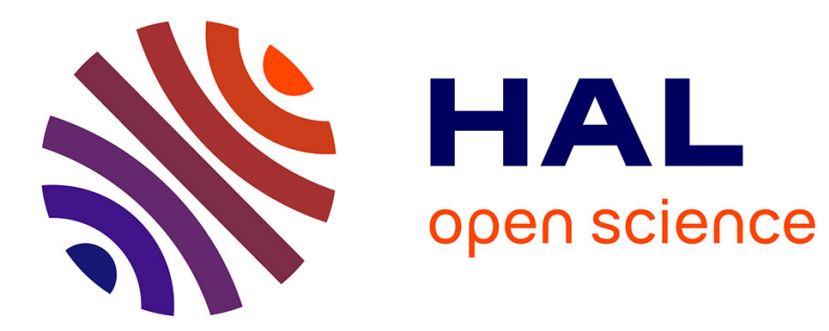

\title{
Design and Control of a Compliant Wheel-on-Leg Rover that Conforms to Uneven Terrain
}

\author{
Arthur Bouton, Christophe Grand, Faïz Ben Amar
}

\section{To cite this version:}

Arthur Bouton, Christophe Grand, Faïz Ben Amar. Design and Control of a Compliant Wheel-on-Leg Rover that Conforms to Uneven Terrain. IEEE/ASME Transactions on Mechatronics, 2020, 25 (5), pp.2354 - 2363. 10.1109/TMECH.2020.2973752 . hal-03019412

\section{HAL Id: hal-03019412 https://hal.sorbonne-universite.fr/hal-03019412}

Submitted on 23 Nov 2020

HAL is a multi-disciplinary open access archive for the deposit and dissemination of scientific research documents, whether they are published or not. The documents may come from teaching and research institutions in France or abroad, or from public or private research centers.
L'archive ouverte pluridisciplinaire HAL, est destinée au dépôt et à la diffusion de documents scientifiques de niveau recherche, publiés ou non, émanant des établissements d'enseignement et de recherche français ou étrangers, des laboratoires publics ou privés. 


\title{
Design and Control of a Compliant Wheel-on-Leg Rover that Conforms to Uneven Terrain
}

\author{
Arthur Bouton, Christophe Grand, and Faïz Benamar
}

\begin{abstract}
This paper presents the design, control and implementation of a compliant wheel-on-leg robot named Complios. This robot is able to spontaneously adapt its configuration while it is rolling on unknown uneven terrain. The contact of all wheels with the ground is constantly ensured by series elastic actuation for each leg. It provides a force control that allows the robot to freely balance its posture while the load can be distributed as equally as possible on each wheel for any relative height differences. The compliant structure of the Complios also gives access to the measurement of horizontal forces applied on legs, which constitutes a valuable information when it comes to choose an appropriate response for obstacle crossing. Finally, the robot is tested on different ground shapes and proves its capability to adapt in real time to the profile.
\end{abstract}

Index Terms-wheel-on-leg robot, compliance, series elastic actuation, posture control, uneven terrain.

\section{INTRODUCTION}

W HEELED locomotion still constitutes the most energyefficient way of moving on flat surfaces. In order to extend these capabilities to uneven terrains, extra degrees of freedom can be added to the frame so as to enable large wheel relative displacements. However, the increase of internal mobility leads to control complexity that has to face the uncertainty regarding the surrounding ground shape.

Indeed, access to a reliable perception and modeling of the ground geometry remains challenging due to sensor noise and inaccuracy of shape interpolation. Though, when planning every movement, even a minor error on ground position invalidates any computed optimization of load distribution. Larger errors can even threat the stability of the whole robot. Some fully actuated wheel-on-leg structures are assisted with local force sensors [1] or torque calculation based on driving current [2], [3] or gear windup [4], but due to the stiffness of these structures, force variations occur in a very narrow range of deviation. Therefore, they have to advance extremely slowly in order to merely maintain wheels in contact with the ground. In addition, stiction in joints can severely interfere with the tracking of force variations [3], [5]. Beyond mapping and wheel placement difficulties, the increasing control complexity entails significant computation times. This has been particularly illustrated during the DARPA Robotics Challenge that ended in June 2015, where many fully capable robots with high physical potential performed so slowly that several teams preferred to avoid the uneven terrain trail because of its cost

A. Bouton and F. Benamar are with Sorbonne Universités, UPMC Univ. Paris 06 and CNRS, UMR 7222, ISIR, F-75005, Paris, France (e-mail: bouton@isir.upmc.fr, amar@isir.upmc.fr).

C. Grand was with ONERA, the French Aerospace Lab, Toulouse, France (e-mail: christophe.grand@onera.fr). in time [6]. Unfortunately, time is often a key factor in robot applications, such as search and rescue robotics [7].

In order to naturally ensure the contact of all wheels with the soil, joints can also be voluntarily let free so as to clear the static indeterminacy. The configuration then conforms to the ground under the effect of gravity. The structure can still include additional actuated joints, so as to keep the control on roll inclination for example [8], [9]. However, free degrees of freedom imply to renounce at least partially to the control of load distribution and stability, which will now depend on the relative wheel height differences.

The proposed approach is then to mix compliance with actuation in order for the structure to spontaneously adapt its configuration to the ground geometry while keeping a control on suspension forces and platform posture. To this end, a specific leg design that emulates an orthogonal decomposition between forces applied on wheels, i.e. between measured horizontal forces and the vertical ones that are controlled, is depicted in section II. The robot posture can then be controlled via an attitude regulator based on a linear inverted pendulum model. This control scheme is described in section III, where wheel speed control is also discussed so as to coordinate the displacements of each leg. In the last section, we present the physical prototype that is tested on different tracks in order to validate the design concept and its control.

\section{Robot Design}

\section{A. Functional Decomposition of Relative Wheel Displace- ments}

In order to devise the desired structure composition, we first identify what main purpose has to be associated with the mobility along each principle directions. In the horizontal direction, wheel positions define the robot size. As the support polygon has to be as large as possible in order to guarantee a good stability against unpredictable disturbances, horizontal wheel positions can be decided at the design stage according to the desired robot size. While maintaining the wheelbase, passive compliance along the longitudinal direction of the robot then proves to be useful when approaching steep obstacles. Indeed, it protects the structure against impact forces, improves the stability during dynamic crossings [10] and helps maintaining good grip with vertical surfaces by avoiding fitful contact losses [11]. Furthermore, if we impose a desired horizontal position for wheels, interaction forces that pressurize the structure are free to be measured. These interaction forces then constitute significant pieces of perceptual information on which to base an additional control for obstacle crossing, as 


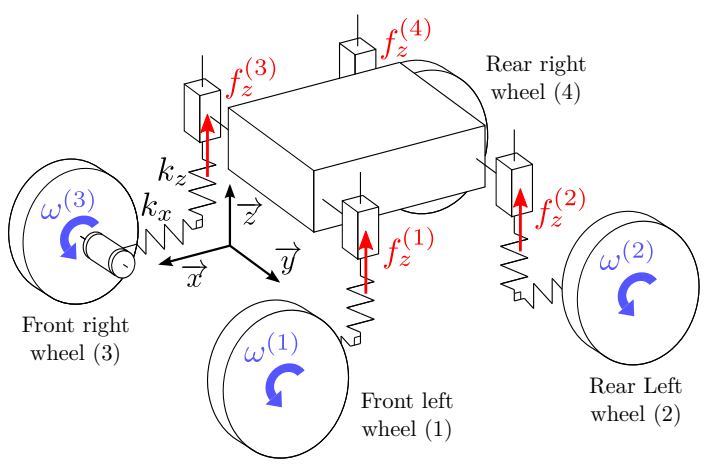

Fig. 1. The ideal decomposition of wheels' relative mobility.

proposed in [12] and [13], where controls for dynamics and quasi-static crossing are addressed.

On the other hand, the vertical position of wheels should adapt to the ground geometry. Instead of a simple passive suspension, differences in wheel elevations should not lead to an uncontrolled imbalance of forces that are supporting the chassis weight. Therefore, a vertical actuation is required in order to provide a combined control of robot stability and load distribution on wheels. When associated with vertical compliance, it forms a series elastic actuator (SEA) [14] that enables a force control rather than a position control. This way, the structure freely adapts leg heights according to the incoming ground variations while the control is focused on regulating vertical forces applied on wheels.

The result is an ideally orthogonal decomposition of wheels' relative mobility and of the corresponding structural stiffnesses, as depicted on Fig. 1. In this model, wheels are horizontally maintained to a reference position by the leg stiffness and the help of wheel speed adjustments, as it will be detailed in section III-B. Conversely, along the vertical direction wheels are free to go up and down while the control focuses on forces applied by legs. Therefore, this analysis respects the force/position orthogonality principle [15].

\section{B. Practical Design}

Thus, the structure design is based on an orthogonal decomposition of mobility that can be theoretically modeled by prismatic joints. However, the use of such joints cannot be reasonably considered in practice because of their vulnerability to cantilever stresses and locking effect when subject to side loads. Indeed, the interaction with ground can lead to high forces in the same plane in which wheels have to be able to move relative to the chassis. Therefore, we choose to use revolute joints, which are both robust to stresses inside the plane of rotation and easy to produce. Provided that it is not in a singular configuration, a kinematic chain comprised of two successive revolute joints suffices to perform the two translations needed in wheel plane. By arranging orthogonally the two lines of this chain which link the wheels to the chassis, the system is placed furthest from singularities while allowing the direct correlation of each joint rotation to a straight translation of the wheel along both perpendicular axes of its plane. In order to achieve the horizontal and vertical

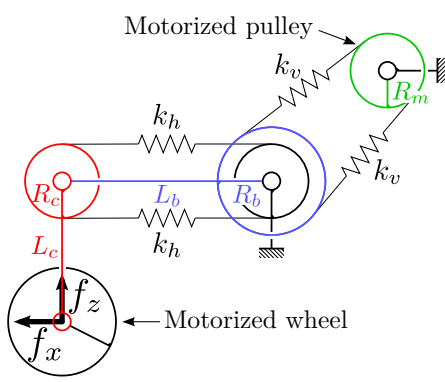

(a) Initial reference position

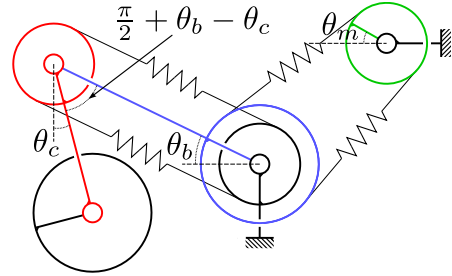

(b) Definition of joint parameters
Fig. 2. Kinematic diagram of one leg. Springs and cables are displayed with thiner black lines, while the reference frame refers to the robot main body.

decomposition along with a maximal wheelbase and ground clearance, the lower segment attached to the wheel have to be vertical while the upper one, linked the chassis, have to be horizontal.

Then, a set of antagonistic springs controls the positioning of each leg segment, as shown on Fig. 2. These springs work in opposition by pulling on each side of pulleys. This way, structural stiffnesses can be exploited on both sides of rest position without exerting alternate stresses on springs if they are sufficiently preloaded. Also, the use of tension springs enables an easier and lighter design because they do not require mechanical guidance. The first set of antagonistic springs, whose stiffness is noted $k_{h}$, tends to maintain the lower segments aligned with the vertical direction of the chassis. This behavior is still true regardless of the leg height, depicted by the angle $\theta_{b}$ at its base, due to the connection of springs to a fixed pulley relative to the chassis. Therefore, for any value of $\theta_{b}$, the leg has a passive stiffness $k_{x}$ along the horizontal direction which can be expressed as a function of the wheel horizontal distance $l_{x}$ from rest position:

$$
k_{x}=2 R_{c}^{2} k_{h} \sqrt{1-\left(\frac{l_{x}}{L_{c}}\right)^{2}}
$$

where $L_{c}$ is the length of the upper segment and $R_{c}$ the radius of the pulley attached to it. A Taylor expansion for $l_{x}$ close to zero then leads to:

$$
k_{x}=2 R_{c}^{2} k_{h}\left(1-\frac{l_{x}^{2}}{2 L_{c}^{2}}+\mathrm{o}\left(l_{x}^{3}\right)\right) .
$$

Thus, the system provides a first order approximation of the horizontal stiffness that is constant around the rest position for any wheel height relative to the chassis.

The second set of springs, with a stiffness $k_{v}$, acts on the upper segment in order to apply the desired torque $\tau_{b}$ at the base of legs. With $\theta_{b}$ and $\theta_{m}$ the angles of the upper segment and the motorized pulley, of respective radius $R_{b}$ and $R_{m}$, the effective torque is:

$$
\tau_{b}=2 R_{b}{ }^{2} k_{v}\left(\frac{R_{m}}{R_{b}} \theta_{m}-\theta_{b}\right) .
$$

So this torque can be controlled according to the SEA principle with a proportional regulator gain $K_{S E A}$, so that: 


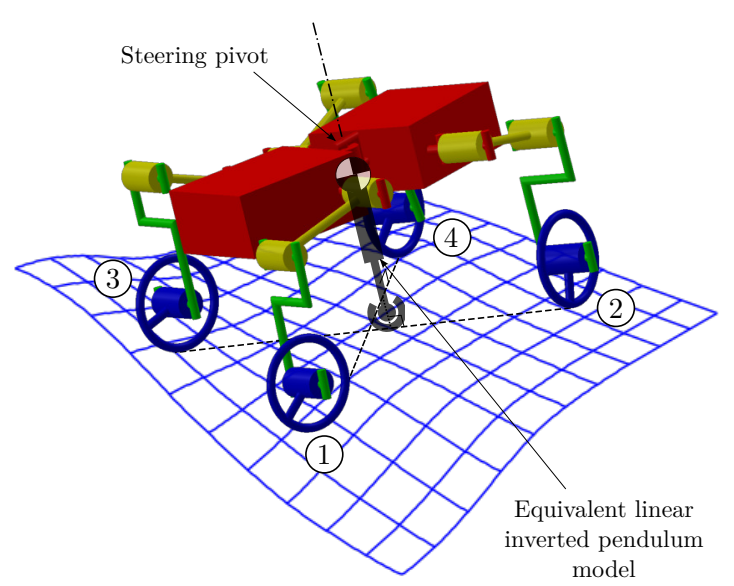

Fig. 3. The overall robot kinematics. Both front and rear bloc of the chassis are in red, wheels are in blue and the linear inverted pendulum to which the system can be equated is shown in black.

$$
\dot{\theta}_{m}=K_{S E A}\left[\tau_{b}^{d}-2 R_{b}^{2} k_{v}\left(\frac{R_{m}}{R_{b}} \theta_{m}-\theta_{b}\right)\right]
$$

where $\tau_{b}^{d}$ is the desired torque.

Then, the vertical force $f_{z}$ applied on a given wheel depends on this torque and is expressed around the initial position by:

$$
f_{z}=\frac{\tau_{b}}{L_{b}} \cos \left(\theta_{b}\right)=\frac{\tau_{b}}{L_{b}}\left(1-\frac{\theta_{b}{ }^{2}}{2}+o\left(\theta_{b}{ }^{3}\right)\right),
$$

with $L_{b}$ the length of the horizontal segment.

Thus, this leg design provides a robust way of approaching the ideal orthogonal decomposition between the different functions we want the structure to perform.

In order to steer, the robot is provided with a revolute joint at the center of the main body that allows both symmetrical front and rear parts of the robot to rotate relative to each other along the vertical axis, as illustrated on Fig. 3. The robot is then theoretically able to turn without any slippage if each wheel speed is properly adapted in accordance with the steering joint angle and rate. With such a design, the path curvature is limited, but extra weight, complexity and weakness of a vertical revolute joint over each wheel is avoided. In addition, pivoting legs as a whole allows the wheels to stay coplanar with the plane in which they are supposed to move relative to the chassis. This way, they can continue to shift horizontally and vertically according to the profile without lateral skidding when the robot turns. Hence, the functional decomposition of movements remains valid at any time.

Moreover, such a steering pivot will play an important role when it comes to negotiate obstacles that block several wheels at a time [16]. As illustrated in [13], it allows the robot to sequence the crossing one wheel after another by modifying their relative equilibrium positions and thus the distribution of horizontal interaction forces with the ground. This way, a strong unique joint can alternatively handle two distinct functions: either the steering or the reconfiguration facing to the obstacles.

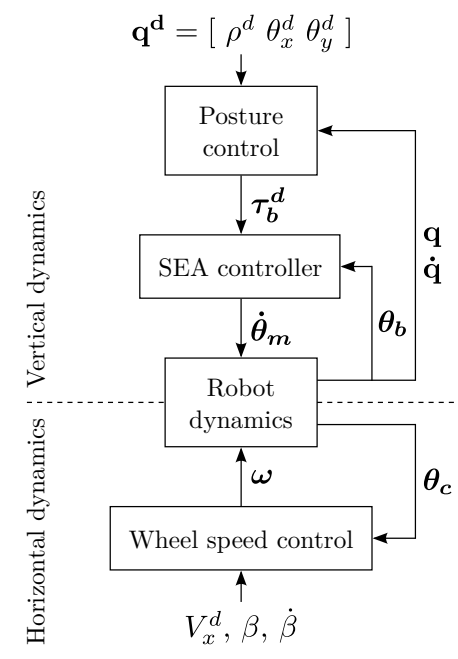

Fig. 4. Overall control diagram.

\section{LEG AND WHEEL CONTROL}

In this section, we address both control of leg torque and wheel speed that allow the robot to conform to uneven terrains while advancing. The overall control scheme is outlined on Fig. 4.

\section{A. Posture Control}

The actuation at leg bases apply a torque $\tau_{b}$ on upper segments, which mainly leads to the control of vertical forces supporting the robot from each side of its center of mass $(\mathrm{CoM})$. The robot posture then depends on the chosen distribution for these torques. In order to coordinate them, we base on an equivalent simplified model. Indeed, the robot standing on parallel legs of variable lengths that always tend to align with the vertical of the chassis can be equated to a linear inverted pendulum. In this model, the ball joint at the base of the pendumlum can be deemed to lay midway between all contact points with the soil, as depicted on Fig. 3. Therefore, the elevation and attitude parameters of the chassis suffice to define its three dimensional pose above the wheels. Let

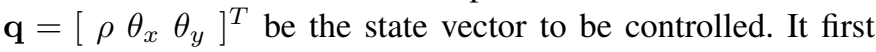
comprises the elevation $\rho$ of the robot CoM from the ground, which is evaluated by the average of vertical wheel distances from leg bases, that is to say:

$$
\rho=\frac{1}{4} \sum_{i=1}^{4}\left(L_{c} \cos \theta_{c}^{(i)}-(-1)^{i} L_{b} \sin \theta_{b}^{(i)}\right) .
$$

The state vector $\mathbf{q}$ also includes both roll and pitch angles of the chassis, respectively noted $\theta_{x}$ and $\theta_{y}$.

Let $\boldsymbol{\theta}_{\boldsymbol{b}}$ and $\boldsymbol{\theta}_{\boldsymbol{c}}$ be the vectors containing respectively the angles $\theta_{b}^{(i)}$ or $\theta_{c}^{(i)}$ of each leg $i$, all defined according to the same transversal direction from left to right. With $\tau_{b}=$ $\left[\tau_{b}^{(1)} \tau_{b}^{(2)} \tau_{b}^{(3)} \tau_{b}^{(4)}\right]^{T}$ the vector consisting of every actuation torque at leg bases, the equations of the main body dynamics can be expressed as:

$\mathbf{M}(\mathbf{q}) \ddot{\mathbf{q}}=\mathbf{B}\left(\boldsymbol{\theta}_{\boldsymbol{b}}, \boldsymbol{\theta}_{\boldsymbol{c}}\right) \tau_{\boldsymbol{b}}+\mathbf{C}\left(\boldsymbol{\theta}_{\boldsymbol{b}}, \boldsymbol{\theta}_{\boldsymbol{c}}\right)+\mathbf{G}(\mathbf{q})+\mathbf{N}(\mathbf{q}, \dot{\mathbf{q}})+\boldsymbol{\eta}$, 
where $\mathbf{M}(\mathbf{q})$ is the inertia matrix, $\mathbf{G}(\mathbf{q})$ expresses the action of gravity, $\mathbf{N}(\mathbf{q}, \dot{\mathbf{q}})$ the action of centrifugal and Coriolis forces and $\boldsymbol{\eta}$ the unknown perturbations or model inaccuracies to be compensated by the controller. In order to match the behavior of the linear inverted pendulum model that best describes the predominant movements of the structure, all moments in (7) are expressed at the center of the ball joint the chassis is assumed to rotate around. This way, we take account of the virtual constraint in dynamics without having to explicitly express forces applied into the ball joint orthogonally to the vertical axis of the chassis, like lateral forces exerted on wheels. In that case, $\mathbf{M}(\mathbf{q})$ and $\mathbf{G}(\mathbf{q})$ are respectively written:

$$
\mathbf{M}(\mathbf{q})=\left[\begin{array}{ccc}
M & 0 & 0 \\
0 & I_{x, G}+M \rho^{2} & 0 \\
0 & 0 & I_{y, G}+M \rho^{2}
\end{array}\right]
$$

and

$$
\mathbf{G}(\mathbf{q})=M g\left[\begin{array}{c}
-\sqrt{\cos \left(\theta_{x}+\theta_{y}\right) \cos \left(\theta_{x}-\theta_{y}\right)} \\
\rho \sin \theta_{x} \\
\rho \sin \theta_{y}
\end{array}\right],
$$

where $M$ is the mass of the chassis, $g$ the norm of gravity acceleration and $I_{x, G}$ and $I_{y, G}$ are both inertia moments of the chassis around respectively its longitudinal and transversal axis at the CoM.

As for $\mathbf{B}\left(\boldsymbol{\theta}_{\boldsymbol{b}}, \boldsymbol{\theta}_{\boldsymbol{c}}\right)$, it expresses the influence of actuation torques on the chassis dynamics, while $\mathbf{C}\left(\boldsymbol{\theta}_{\boldsymbol{b}}, \boldsymbol{\theta}_{\boldsymbol{c}}\right)$ gives the effect of stresses generated by the elasticity in passive joints.

According to the robot kinematics, while neglecting leg masses and wheel torques, the $i$ th column of $\mathbf{B}\left(\boldsymbol{\theta}_{\boldsymbol{b}}, \boldsymbol{\theta}_{\boldsymbol{c}}\right)$, which corresponds to the action of leg $i$, is expressed at the center of the virtual ball joint by:

$$
\begin{aligned}
& \mathbf{B}^{(i)}\left(\boldsymbol{\theta}_{\boldsymbol{b}}, \boldsymbol{\theta}_{\boldsymbol{c}}\right)=\left[\begin{array}{c}
0 \\
0 \\
-1
\end{array}\right] \\
& +\frac{\cos \theta_{c}^{(i)}}{L_{b} \cos \left(\theta_{b}^{(i)}-\theta_{c}^{(i)}\right)}\left[\begin{array}{c}
-(-1)^{i} \\
(-1)^{\frac{i(i+1)}{2}+i} L_{y} \\
-L_{x}-(-1)^{i} \rho \tan \theta_{c}^{(i)}
\end{array}\right],
\end{aligned}
$$

when leg indexes are counted in this order: front left, rear left, front right and rear right, as described on Fig. 1. Under the same assumptions, $\mathbf{C}\left(\boldsymbol{\theta}_{\boldsymbol{b}}, \boldsymbol{\theta}_{\boldsymbol{c}}\right)$ is written:

$$
\begin{aligned}
& \mathbf{C}\left(\boldsymbol{\theta}_{\boldsymbol{b}}, \boldsymbol{\theta}_{\boldsymbol{c}}\right)=\sum_{i=1}^{4} 2 R_{c}{ }^{2} k_{h} \theta_{c}^{(i)}\left(\left[\begin{array}{l}
0 \\
0 \\
1
\end{array}\right]\right. \\
& \left.+\frac{\sin \theta_{b}^{(i)}}{L_{c} \cos \left(\theta_{b}^{(i)}-\theta_{c}^{(i)}\right)}\left[\begin{array}{c}
1 \\
-(-1)^{\frac{i(i+1)}{2}} L_{y} \\
(-1)^{i} L_{x}-\frac{\rho}{2} \sin \frac{\theta_{b}^{(i)}}{2}
\end{array}\right]\right) .
\end{aligned}
$$

The robot posture can then be controlled with respect to the chassis dynamics through a proportional-derivative controller, as expressed below:

$$
\begin{array}{r}
\tau_{\boldsymbol{b}}=\mathbf{B}\left(\boldsymbol{\theta}_{\boldsymbol{b}}, \boldsymbol{\theta}_{\boldsymbol{c}}\right)^{+}\left[\mathbf{M}(\mathbf{q})\left(\mathbf{K}_{\mathbf{p}} \widetilde{\mathbf{q}}-\mathbf{K}_{\mathbf{d}} \dot{\mathbf{q}}\right)\right. \\
\left.-\mathbf{C}\left(\boldsymbol{\theta}_{\boldsymbol{b}}, \boldsymbol{\theta}_{\boldsymbol{c}}\right)-\mathbf{G}(\mathbf{q})\right],
\end{array}
$$

where $\widetilde{\mathbf{q}}=\mathbf{q}_{\mathbf{d}}-\mathbf{q}$ is the difference with the desired state vector $\mathbf{q}_{\mathbf{d}}$ and $\mathbf{K}_{\mathbf{p}}$ and $\mathbf{K}_{\mathbf{d}}$ are the diagonal matrices of respectively proportional and derivative gains. We here neglect the centrifugal and Coriolis forces, that can be considered as perturbations to be rejected.

$\mathbf{B}^{+}$is then the pseudo-inverse of $\mathbf{B}$, giving the solution $\tau_{b}$ that minimizes the quadratic sum of all applied torques from SEA. As the robot stands on four legs while there is three independent variables to be controlled, there is one degree of freedom in the choice of the actuation distribution. With the pseudo-inverse matrix, we thus obtain the less consuming distribution that achieves the desired effect on chassis dynamics. This distribution can then be modulated along the kernel of $\mathbf{B}$, which can be given by a singular value decomposition, with no influence on the resulting posture control. This allows the robot to increase the pressure on two diagonally opposed wheels while the load on both other wheels is mitigated in order to enable the crossing of steepest obstacles when friction coefficient is not large enough.

As the legs are symmetrically distributed around the CoM, an horizontal attitude of the chassis leads to an equal distribution of the load on wheels when their torques are negligible. Then, a balanced load ensures the best stability against unforeseen perturbations from any direction and allows the robot to recruit a well distributed amount of traction from all wheels while lowering slippage. On soft soils, it also maximizes the obtainable traction by limiting the sinkage of each wheel. Therefore, it can be deemed to be the less consuming configuration for both leg and wheel actuation when all wheels are in the same grip condition with the ground. In addition, this configuration helps maintaining the distinction between the horizontal measured forces, which indicate the presence of obstacles, and the vertical controlled forces, related to the load distribution. For all these reasons, the default desired attitude is the horizontal one, i.e. with $\theta_{x}=0$ and $\theta_{y}=0$. The desired elevation $\rho$ is then defined by the reference configuration of legs when both of their segments are orthogonal. This last value is thus determined by the length $L_{c}$ of vertical segments, chosen at design stage according to a compromise between stability and ground clearance.

Also, it is possible to change the position of the CoM relative to the wheels by simply modifying the desired chassis attitude. Indeed, while the robot tilts away from the vertical, the CoM is moved in the same direction according to the inverse pendulum model. This can be used to modify the load distribution on legs and even to be able to sustain on only three wheels. An exploitation of both the modulation of actuation distribution and CoM displacement for obstacle crossing can be found in [13].

\section{B. Wheel Speed Control}

Each wheel speed has to be adapted according to the steering joint angle and rotation speed in order to avoid wheel 


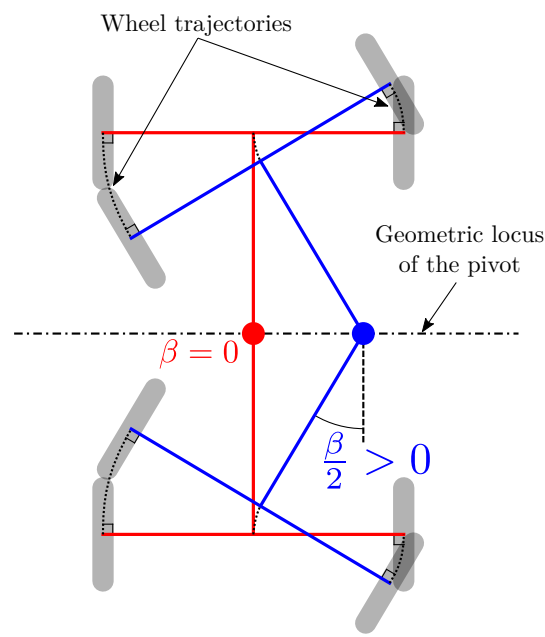

Fig. 5. Rotation of the steering pivot without wheel slip while the robot is at a standstill. The trajectories of wheels and steering pivot are depicted from a top view.

slippage and internal stresses while turning. If the robot stands still on a flat surface and rotates its chassis, the transverse plane should be preserved as a symmetry plane, which gives us the geometric locus of the steering pivot relative to the chassis frame, as shown on Fig. 5. Then, by imposing no lateral wheel velocity when the steering pivot moves along its locus, we find that the speed of wheel $i$ should be:

$$
\omega_{\text {steer }}^{(i)}=\left((-1)^{i} L_{x} \tan \frac{\beta}{2}-(-1)^{\frac{i(i+1)}{2}} L_{y}\right) \frac{\dot{\beta}}{2 R},
$$

where $\beta$ is the steering angle between front and rear parts of the chassis, defined positive when heading to the left. $L_{x}$ and $L_{y}$ are then respectively the longitudinal and transversal distance of wheel positions from the steering pivot, while $R$ is the wheel radius.

For a given steering angle, left and right wheels then require a speed differential for the robot to advance at a desired tangential velocity $V_{x}^{d}$ without slipping:

$$
\Delta \omega_{\text {turn }}^{(i)}=(-1)^{\frac{i(i+1)}{2}} \frac{L_{y}}{L_{x}} \tan \left(\frac{\beta}{2}\right) \frac{V_{x}^{d}}{R} .
$$

Therefore, due to the superposition of velocities, the robot can freely roll and turn on a flat ground with theoretically no slippage if wheels observe the sum of speed variations $\omega_{\text {steer }}$ and $\Delta \omega_{\text {turn }}$.

However, when the robot advances on arbitrary ground shapes, wheels are shifted from their reference position due to slope differences, as illustrated on Fig. 6a. Enabled by the leg compliance, these relative displacements in wheel horizontal positions lead to persisting internal stresses that can be indefinitely held by ground friction, even after getting back on a flat ground, as shown on Fig. 6b. This is why we add a proportional wheel speed modulation in accordance with the angular deviation $\theta_{c}$ of each vertical leg segment from its rest position.

Thus, the desired speed for wheel $i$ is:

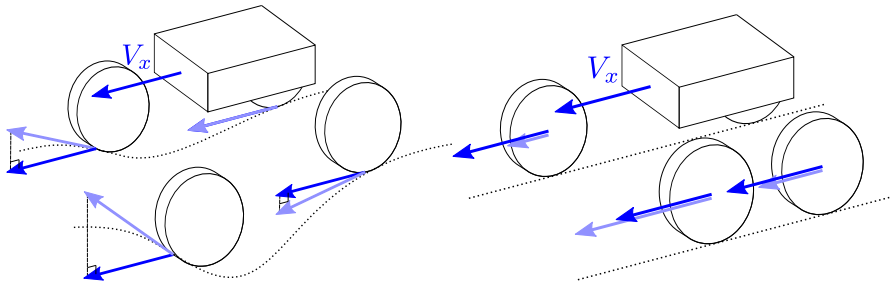

(a) Speed differentials on slopes

(b) Relaxation of constraints

Fig. 6. Illustration of the need for wheel speed adaptation. Darker arrows corresponds to the chassis horizontal velocity, while brighter ones stand for the adapted wheel velocities.

$$
\omega^{(i)}=\frac{V_{x}^{d}}{R}+\omega_{\text {steer }}^{(i)}+\Delta \omega_{\text {turn }}^{(i)}+\frac{\left|V_{x}^{d}\right|}{R} k_{\omega} \theta_{c}^{(i)},
$$

where $k_{\omega}$ is the proportional gain of the speed regulator.

While it assists the horizontal wheel positioning, this control therefore allows the robot to adjust the speed of wheels that are rolling on different slopes than the average plane of contacts with the ground.

\section{EXPERIMENTAL REALIZATION}

In this section, we describe the practical implementation of the suggested locomotion structure. In particular, we address here how the legs can passively compensate for the robot's weight and what are the criteria for the choice of spring stiffnesses. The electronic architecture of the robot is then exposed. Finally, the robot is tested on several ground profiles.

\section{A. Mechanical Design}

The Complios, that is shown on Fig. 7, has a nominal height of $34 \mathrm{~cm}$ and weighs about $15 \mathrm{~kg}$. It stands on four wheels of $15 \mathrm{~cm}$ diameter, with a $65.5 \mathrm{~cm}$ wheelbase and a $38 \mathrm{~cm}$ track. The whole robot consists of four symmetrical subassemblies operating each leg independently.

1) Layout of Main Elements: As it can be seen on Fig. 8, the framework of leg segments is made of parallel aluminium plates that are paired by spacers long enough to let the springs fit in the gap while ensuring a good flexural strength against lateral forces. Spring cables are attached to bilateral pulleys printed in polyamide and capable of adjusting the relative cable tension. These pulleys transmit the torque whether to the lower leg segment or to the SEA shaft. The aluminium pulley between plates at leg base is mounted on a ball bearing because it is guiding the $k_{h}$ spring cables that are tied to the chassis, so that it can stand still while the shaft is rotating at its center.

Motors that operate the legs are disposed at both front and rear sides of the chassis. An additional mechanism in charge of relieving motors from the weight of the robot is housed in the remaining space between SEA springs. This way, robot size does not exceed the volume imposed by legs in the reference configuration. 


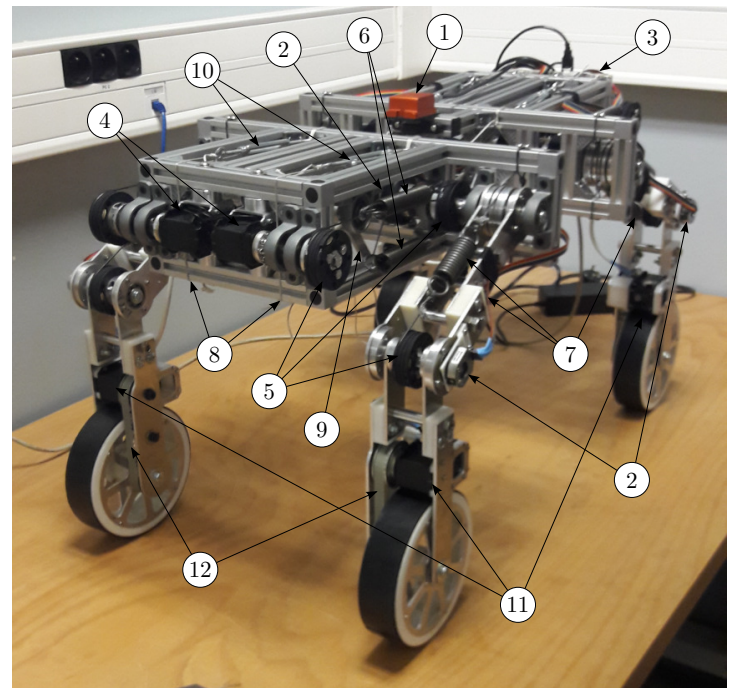

(1) Inertial measurement unit

(2) Absolute encoders

(3) Embedded computer

(4) Digital servomotors dedicated to leg actuation

(5) Bilateral pulleys

(6) Springs that form the series elastic actuation

(7) Retaining springs of the vertical segments

(8) Cables connected to the weight compensation springs

(9) Lever of the weight compensation mechanism

(10) Tensioners of the weight compensation springs

(11) Digital servomotors dedicated to wheel traction

(12) Drive belts

Fig. 7. The Complios robot from a three-quarters view.

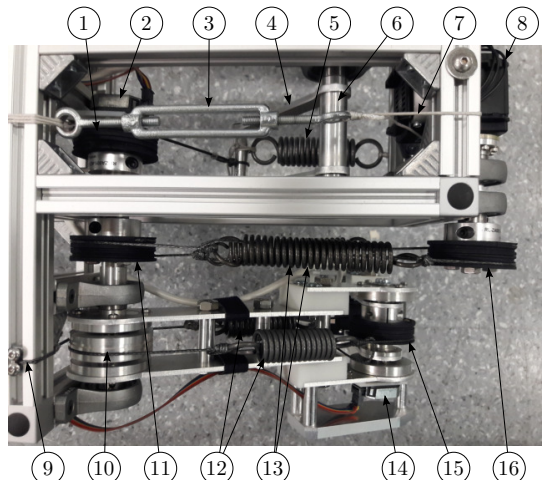

(1) Pulley of the $\mathrm{WCM}^{*}$

(2) Encoder measuring $\theta_{b}$

(3) Tensioner of the WCM

(4) Lever of the WCM

(5) Spring of the WCM

(6) Lever axis

(7) Cable of the WCM spring

(8) Motor of leg actuation

(9) Anchoring of the lower sprin

(10) Free pulley on ball bearings

(11) $R_{b}$ pulley

(12) $k_{h}$ springs

(13) $k_{v}$ springs

(14) Encoder measuring $\theta_{b}-\theta_{c}$

(15) $R_{c}$ pulley

(16) $R_{m}$ pulley

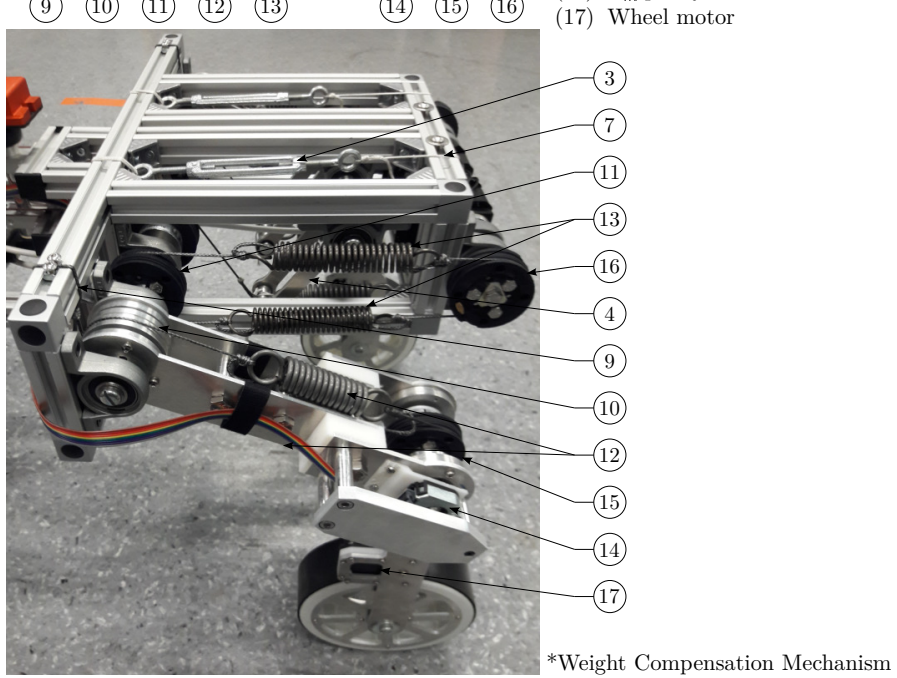

Fig. 8. Focus on a quarter robot from top and high-angle views.

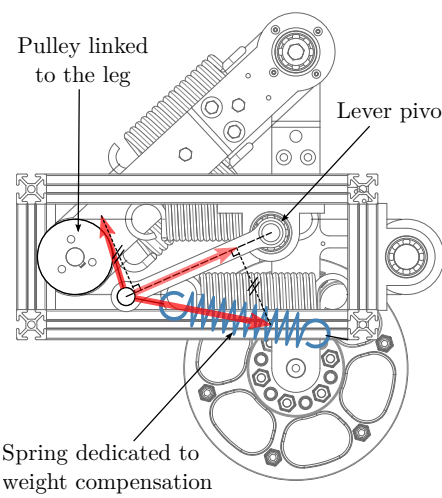

(a) Leg in upper position

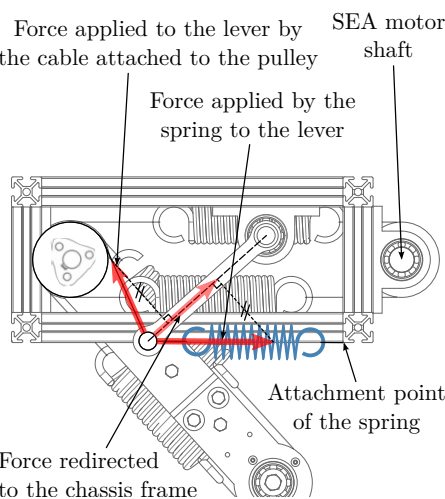

(b) Leg in lower position
Fig. 9. Description of the weight compensation mechanism. These transverse sectional views depict two extremal leg configurations for which the produced pushing force is equal.

2) Weight Compensation: Legs have to continuously support the chassis' weight. Around the desired reference posture, for which the load is well balanced, it means that each leg base shaft have to sustain a torque of about the quarter of the chassis' weight times the length $L_{b}$ of upper segments. In order to avoid the actuation from generating such a torque all the time, we add a passive mechanism acting on leg shaft in parallel with SEA. Based on the use of preloaded springs, it aims at producing the proper counterweighting torque, regardless of the leg vertical position which is correlated with its shaft position. Springs with the lowest stiffness minimize the effect of length variations on the resulting torque. However, it would require a significant extension or compression, according to the type of spring used, in order to reach the required preload. As it can not be done to a large extent in practice, we propose to help homogenize the force along the range of leg displacements with an intermediary lever.

This lever, simple to implement, redirects a part of the force produced by springs to the rigid frame of the chassis, as depicted on Fig. 9. While the leg rotates, the angle of the lever changes. As a consequence, the proportion of transmitted force from the spring to the pulley changes as well. Therefore, the force alteration from spring length variations can be compensated by the modulation of the amount of force absorbed in the lever.

The geometric parameters of the mechanism have been optimized by an exhaustive research within the size limits of the chassis so as to minimize the deviation of the vertical force applied at the end of the leg throughout its range of vertical displacement. As shown on Fig. 10, the obtained mechanism substantially reduces the work of actuation when legs move away from their initial position.

As it is visible on Fig. 8, the preload of springs dedicated to weight compensation can be adjusted through tensioners that are easily accessible from the top of the robot, while tension cables are running around the chassis, beside motor shafts.

3) Spring Selection: Theoretically, in order to obtain the most responsive force tracking with a finite velocity source of actuation, SEA springs should be as stiff as possible. Indeed, a higher stiffness leads to larger modifications of force for a 


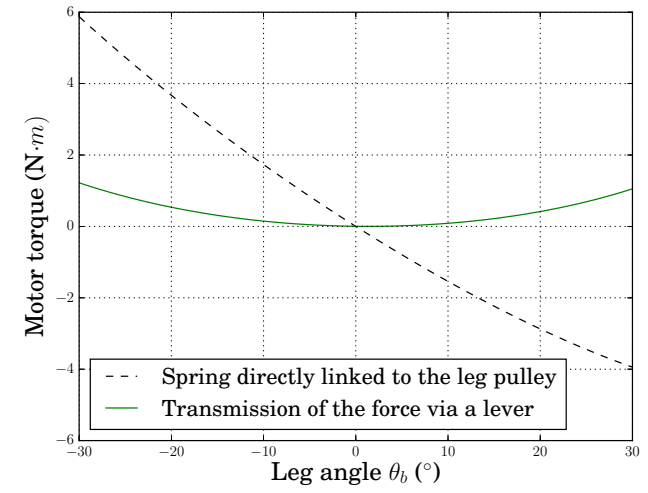

Fig. 10. Comparison of required motor torques in order to counterbalance the robot's weight depending on the spring attachment. In both case, preload of the $15 \mathrm{~N} / \mathrm{mm}$ spring is set so as to exactly compensate the weight when the robot is in the initial reference configuration.

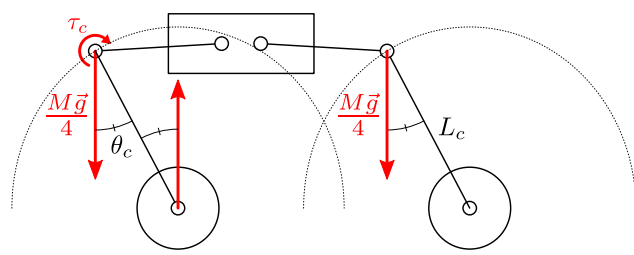

Fig. 11. Fall of the robot in the sagittal plane.

same variation of length. However, four criteria narrow this stiffness:

- Natural frequencies of the whole passive system, consisting of the chassis standing on the four compliant legs, have to lie within the bandwidth of actuation, so they can be actively absorbed. This has to be verified for the three main excitation modes, namely vertical chassis translations and robot swings along both roll and pitch axis.

- Forces transmitted by SEA springs must not threat the actuation when legs are subject to sudden height variations. Thus, the spring stiffness has to be low enough so as to protect the motorization against sharp leg displacements induced by changes in ground geometry that are too fast for the regulator to adapt SEA position in time.

- The resolution of torque evaluation, inversely proportional to the spring stiffness, have to be high enough in order to result in a fluid SEA control with no quantification noise.

- Internal stresses caused by the required preload of antagonistic springs, so as to ensure their constant tension over the full range of displacements, have to meet the resistance limits of the chassis' structure.

In the case of our prototype, the last criterion was the most restrictive because an increase of internal stresses would have require stronger materials, resulting in an increase of the total weight of the system.

For the horizontal compliance, a lower limit also appears in the choice of spring stiffness. Indeed, the kinematics of the robot allows it to fall forwards or backwards in a circular trans-

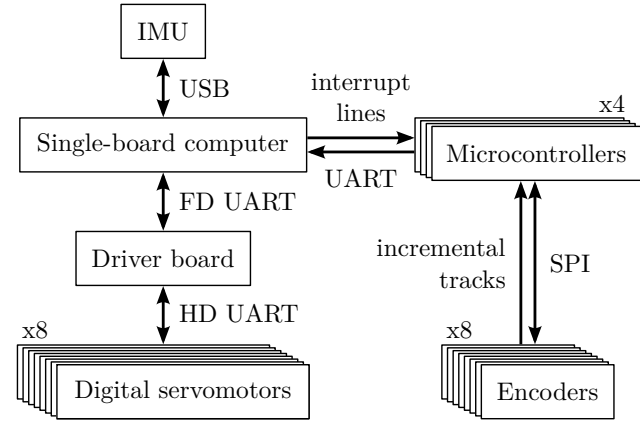

Fig. 12. Diagram of the Complios electronic architecture.

lation movement, as shown on Fig. 11. Therefore, we must ensure that the torque generated by $k_{h}$ springs is sufficient to maintain the robot upright. By statically isolating the leg lower segments, we deduce that the initial reference configuration is a stable equilibrium if and only if for any angle $\theta_{c}$ :

$$
\operatorname{sgn}\left(\theta_{c}\right) 2 R_{c}^{2} k_{h} \theta_{c}>\operatorname{sgn}\left(\theta_{c}\right) \frac{M g}{4} L_{c} \sin \theta_{c}
$$

For this to be always true, according to the derivative at zero, $k_{h}$ has thus to verify:

$$
k_{h}>\frac{M g L_{c}}{8 R_{c}^{2}}
$$

Hence, stiffnesses of $4.27 \mathrm{~N} / \mathrm{mm}$ for $k_{v}$ and $15 \mathrm{~N} / \mathrm{mm}$ for $k_{h}$ have been chosen for our prototype.

\section{B. Electronic Architecture}

The electronic architecture of the robot is depicted on Fig. 12. Controls presented in III are managed by an embedded single-board computer based on a AM3358 processor with a $1 \mathrm{GHz}$ clock. The posture control loop is then running at the frequency of $40 \mathrm{~Hz}$ on a Real-Time Linux kernel. The chassis attitude is provided by an IMU that includes a Kalman filter merging inertial data with geomagnetic field measurements. Angular positions of leg joints are given by two absolute encoders per leg with a resolution of 4096 points per revolution. A microcontroller clocked at $72 \mathrm{MHz}$ is dedicated to each leg and is in charge of following the absolute angular positions via both an SPI communication with the encoders and a tracking of their incremental signals. Then, position data are transmitted to the central computing unit via UART when an interrupt signal is triggered to the microcontrollers, so that the control of response times can be guaranteed at each request. The position of motor shafts, for their part, are measured by absolute encoders included in the digital servomotors and inquired via the same UART channel that is used for delivering speed setpoints. Main characteristics of these servomotors are given by Table I.

\section{Testing Results}

The robot is tested on an asymmetric terrain where left wheels have to cross a trapezoidal obstacle as high as wheel diameter, i.e. $15 \mathrm{~cm}$. Both ascending and descending slopes 


\begin{tabular}{|c|c|c|}
\cline { 2 - 3 } \multicolumn{1}{c|}{} & Leg actuation & Wheel actuation \\
\hline Gear ratio & $225: 1$ & $200: 1$ \\
\hline Stall torque & $8.4 \mathrm{~N} \mathrm{~m}$ & $6 \mathrm{~N} \mathrm{~m}$ \\
\hline No load speed & $45 \mathrm{RPM}$ & $63 \mathrm{RPM}$ \\
\hline
\end{tabular}

TABLE I

CHARACTERISTICS OF THE SERVOMOTORS USED
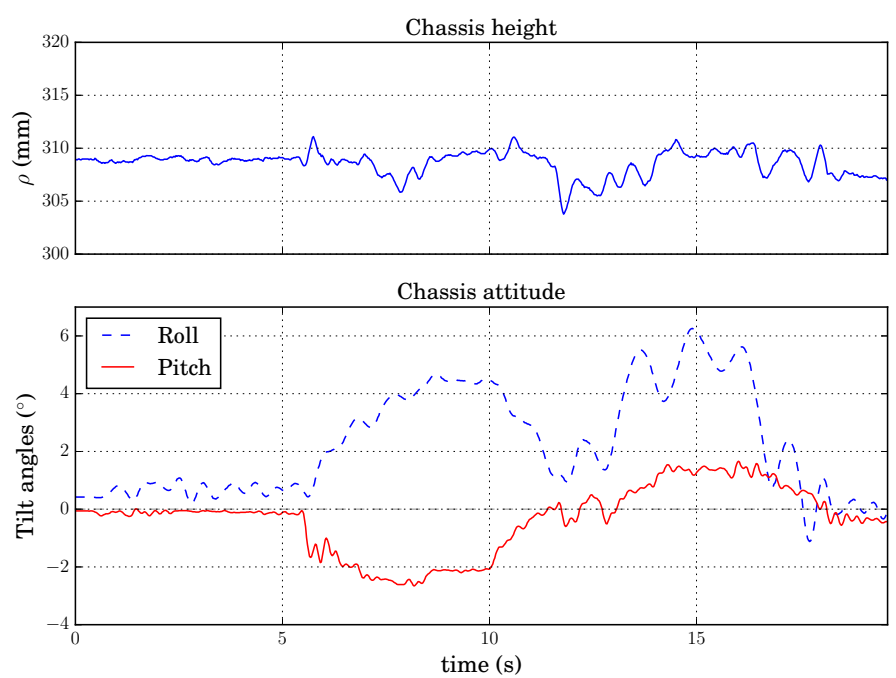

Fig. 13. Posture variations during the crossing of a trapezoidal obstacle.

are $30^{\circ}$, so that a balanced load on wheels is still suitable to climb the obstacle. While the robot moves forward at $0.1 \mathrm{~m} / \mathrm{s}$, snapshots of the crossing can be seen on Fig. 15 and the chassis attitude and elevation during the experiment are shown on Fig. 13. We observe that the roll and pitch angles barely exceed $6^{\circ}$, while the elevation remains confined into a $8 \mathrm{~mm}$ extent. On Fig. 14, we verify the relative leg length adaptation as the robot advances on the obstacle. Thus, the trapezoidal shape appears successively on leg 1 and 2 , while the other legs retract in order to maintain constant then elevation of the chassis.

Furthermore, leg deformations give a representation of in-
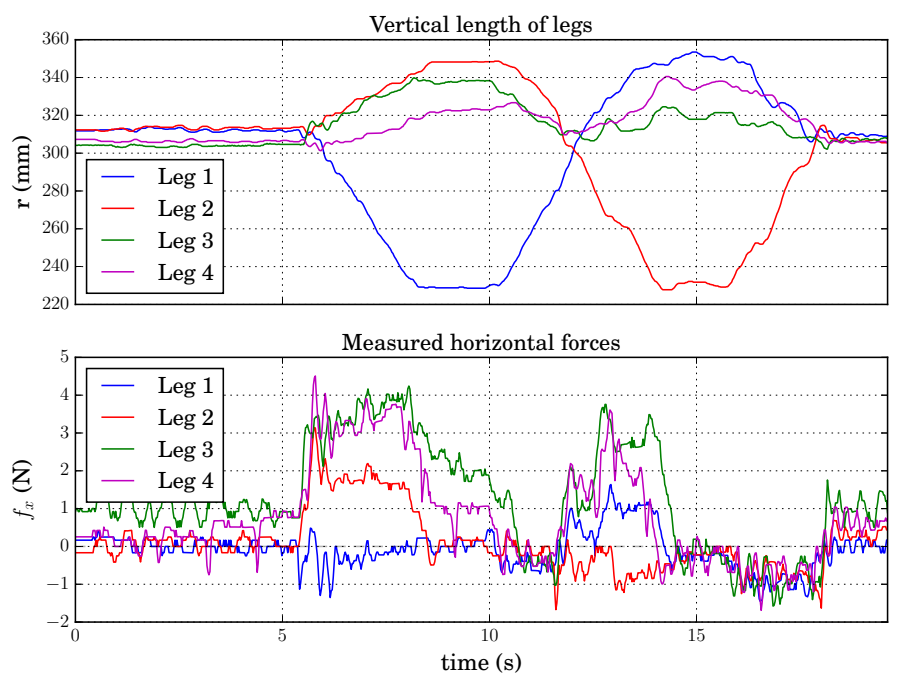

Fig. 14. Leg deformations during the crossing of a trapezoidal obstacle.

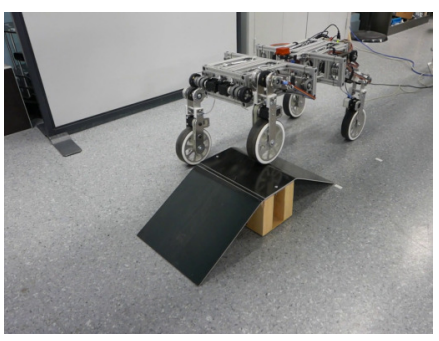

(a)

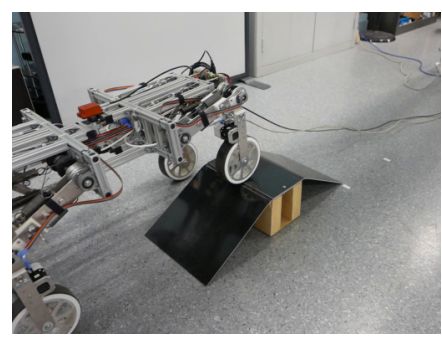

(b)
Fig. 15. Crossing of a trapezoidal obstacle. Video can be found at http: //www.isir.upmc.fr/vid/complios_terrain_1.mp4.

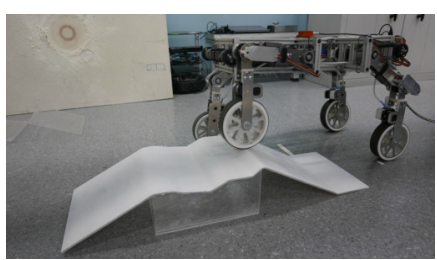

(a)

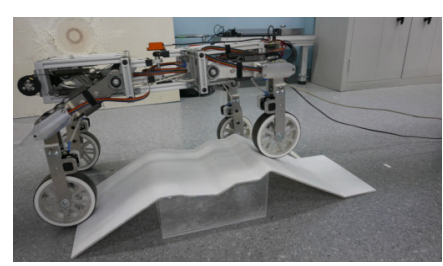

(b)
Fig. 16. Crossing of an arbitrary profile (http://www.isir.upmc.fr/vid/ complios_terrain_2.mp4).

ternal stresses caused by the ground pressuring the structure. Indeed, while neglecting the leg masses and wheel torques, horizontal force applied on leg $i$ can be expressed by:

$$
f_{x}^{(i)}=\frac{-(-1)^{i} L_{c} \sin \theta_{c}^{(i)} \tau_{b}^{(i)}+L_{b} \cos \theta_{b}^{(i)} \tau_{c}^{(i)}}{L_{b} L_{c} \cos \left(\theta_{b}^{(i)}-\theta_{c}^{(i)}\right)}
$$

We then notice an orderly dispersion of these forces when wheels are in contact with the ascending slope. The observation of these forces can therefore be used for the characterization of the encountered difficulties and then to determine how to modify the load distribution in order to cross the steepest obstacles, as examined in [13].

The crossing of two other terrains, with different profiles and surface conditions can be observed on Fig. 16 and 17. The last one proves in particular the robot ability to keep balance in the presence of serious wheel slips, while climbing a $50^{\circ}$ slope.

\section{CONCLUSION}

The Complios stands on four wheels that are each linked to the chassis via a compliant leg. These legs are comprised

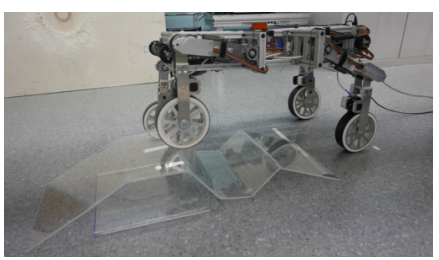

(a)

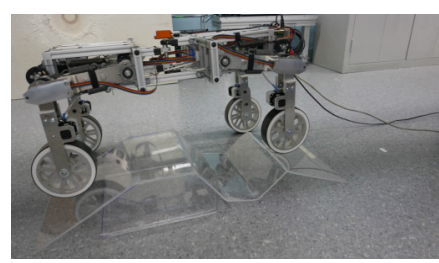

(b)
Fig. 17. Crossing of a terrain including a basin (http://www.isir.upmc.fr/vid/ complios_terrain_3.mp4). 
of two pivoting segments subject to the action of antagonistic springs. A series elastic actuation then allows the Complios to balance while controlling the vertical forces applied on wheels. Therefore, an even distribution of the load can be ensured in order to maximize the achievable traction and stability of the robot in most situations. The structure is also design so as to distinguish the horizontal forces induced by the presence of surrounding obstacles.

The Complios design enables the application of crossing responses to steep obstacles without requiring any prior knowledge on ground geometry or complex heavy planning, as describe in [12] or [13].

\section{REFERENCES}

[1] C. Grand, "Optimisation et commande des modes de déplacement des systèmes locomoteurs hybrides roue-patte: application au robot hylos," Ph.D. dissertation, Paris 6, 2004.

[2] S. Yleonen and A. Halme, "Further development and testing of the hybrid locomotion of w orkpartner robot," in Proc. 5th International Conference on Climbing and Walking Robots (CLAWAR2002), 2002, pp. 293-298.

[3] C. Grand, P. Jarrault, F. B. Amar, and P. Bidaud, "Experimental evaluation of obstacle clearance by a hybrid wheel-legged robot," in Experimental Robotics. Springer, 2016, pp. 47-58.

[4] B. H. Wilcox, T. Litwin, J. Biesiadecki, J. Matthews, M. Heverly, J. Morrison, J. Townsend, N. Ahmad, A. Sirota, and B. Cooper, "Athlete: A cargo handling and manipulation robot for the moon," Journal of Field Robotics, vol. 24, no. 5, pp. 421-434, 2007.

[5] P. Jarrault, "Optimisation des capacités de franchissement des robots mobiles hybrides" roues-pattes"," Ph.D. dissertation, Paris 6, 2013.

[6] S. Karumanchi, K. Edelberg, I. Baldwin, J. Nash, J. Reid, C. Bergh, J. Leichty, K. Carpenter, M. Shekels, M. Gildner, et al., "Team robosimian: Semi-autonomous mobile manipulation at the 2015 darpa robotics challenge finals," Journal of Field Robotics, vol. 34, no. 2, pp. 305-332, 2017.

[7] R. R. Murphy, S. Tadokoro, D. Nardi, A. Jacoff, P. Fiorini, H. Choset, and A. M. Erkmen, "Search and rescue robotics," in Springer Handbook of Robotics. Springer, 2008, pp. 1151-1173.

[8] K. Iagnemma, A. Rzepniewski, S. Dubowsky, P. Pirjanian, T. Huntsberger, and P. Schenker, "Mobile robot kinematic reconfigurability for rough-terrain," in Proc. SPIE, vol. 4196, 2000, pp. 413-420.

[9] D. Wettergreen, S. Moreland, K. Skonieczny, D. Jonak, D. Kohanbash, and J. Teza, "Design and field experimentation of a prototype lunar prospector," The International Journal of Robotics Research, vol. 29, no. 12, pp. 1550-1564, 2010.

[10] J. Fauroux, J. Dakhlallah, and B. Bouzgarrou, "A new concept of fast mobile rover with improved stability on rough terrain," Proc. of HUDEM, 2010.

[11] A. K. Singh, R. K. Namdev, V. Eathakota, and K. M. Krishna, "A novel compliant rover for rough terrain mobility," in Intelligent Robots and Systems (IROS), IEEE/RSJ Int. Conf. on, 2010, pp. 4788-4793.

[12] A. Bouton, C. Grand, and F. Benamar, "Motion control of a compliant wheel-leg robot for rough terrain crossing," in Robotics and Automation (ICRA), 2016 IEEE International Conference on. IEEE, 2016, pp. 2846-2851.

[13] _ "Obstacle negotiation learning for a compliant wheel-on-leg robot," in Robotics and Automation (ICRA), 2017 IEEE International Conference on. IEEE, 2017, pp. 2420-2425.

[14] G. A. Pratt and M. M. Williamson, "Series elastic actuators," in Intelligent Robots and Systems (IROS), IEEE/RSJ Int. Conf. on, 1995.

[15] L. Villani and J. De Schutter, "Force control," in Springer Handbook of Robotics, 2008, pp. 161-185.

[16] J.-C. Fauroux, F. Chapelle, and B. Bouzgarrou, "A new principle for climbing wheeled robots: Serpentine climbing with the open wheel platform," in Intelligent Robots and Systems, 2006 IEEE/RSJ International Conference on. IEEE, 2006, pp. 3405-3410.

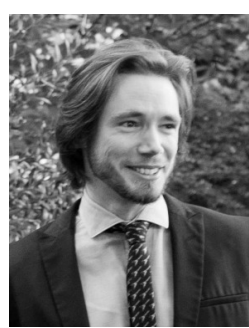

Arthur Bouton is graduated from the École Nationale Supérieure d'Arts et Métiers ParisTech (France) and has a Ph.D. degree from Sorbonne Universités, UPMC University Paris 06 (France). His Ph.D. dissertation was on the design and control of a compliant locomotion structure for obstacle crossing, with the supervision of Faïz Ben Amar and Christophe Grand. Since 2016, he has been a teaching and research assistant for Sorbonne Universités in charge of teaching control theory, machine learning and robotic control.

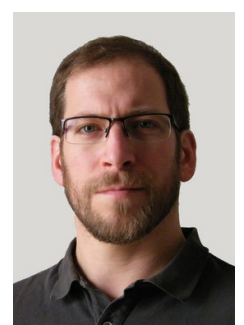

Christophe Grand received his M. Sc. degrees in mechanical engineering from ENSAM, Paris, France in 2000 and the $\mathrm{Ph}$. D. degrees in Robotics from the University Pierre et Marie Curie (UPMC), Paris, France in 2004. From 2005 to 2013, he was associate professor at UPMC, in the Robotics Department. Since 2013, he is a research follow at ONERA, in the Information Processing and System Department. His research interest covers robotics, embedded systems and multi-robot cooperation.

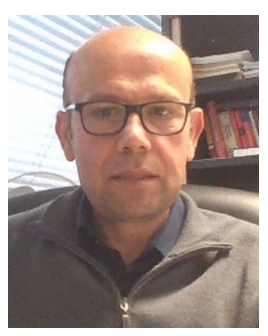

Faz Ben Amar received the Graduate degree, in 1990, from the École Nationale Supérieure des Arts et Métiers, of Paris (France), a Master and Ph.D. in Robotics from University Pierre et Marie Curie in 1994. He was associate professor at University of Versailles and now he is full professor at Sorbonne Université (formerly Université Pierre et Marie Curie UPMC). He is the team leader of the SyRoCo group attached to the ISIR (Institute of Intelligent Systems and Robotics) and the Co-director of the Engineering Doctoral School at Sorbonne Université. His research concerns land and marine mobile robotics, design and control of high mobility agile robots, modular robotics, navigation in unstructured environment, vehicle dynamics, humanoid postural balance and walking. 